\title{
Many Traditions, One Practice Tieraona Low Dog, M.D. - A Modern Eclectic
}

\section{Russ Mason, M.S.}

\section{Introduction}

$\mathbf{W}$ hen I began to interview Tieraona Low Dog, M.D., I could not have foreseen that her story-quite apart from her important views on integrative medicine and her numerous honors and achievements-had its true genesis in a life-defining moment when she was 19 years old. People who write screenplays call such an occurrence "the inciting incident" and Dr. Low Dog's inciting incident is every bit as dramatic as one finds in the movies.

Dr. Low Dog is an assistant clinical professor in the department of family and community medicine, at the University of New Mexico School of Medicine, Albuquerque. She is also the medical director of the TreeHouse Center of Integrative Medicine, also in Albuquerque.

Dr. Low Dog received her Doctor of Medicine degree from the University of New Mexico School of Medicine Albuquerque, after many years of practicing as an herbalist. She has published numerous articles on women's health, including papers on integrative approaches to premenstrual syndrome, menopause, and breast cancer, and a safety review of black cohosh (Cimicifuga racemosa; see box entitled How Safe is Black Cohosh? Dr. Low Dog's Integrative Approach to the Data). Dr. Low Dog has also copublished on the topic of herb usage among Hispanic elders.

In 1998, she was a recipient of the Martina de la Cruz Medal for her work with indigenous people and remedies. She is a highly sought-after speaker for medical conferences and community groups to address topics ranging from the cultural aspects of alternative medicine to current scientific information on herbs sold in the United States. As one of the country's leading experts on botanical and integrative medicine, Dr. Low Dog was chosen to be a member of the White House Commission on Complementary and Alternative Medicine Policy.

\section{The Interview}

Russ Mason: You are perhaps best known for your work on integrating botanicals into mainstream medical practice. Is this a fair assessment?

Tieraona Low Dog: It's somewhat of a mischaracterization to say that I am only promoting integration of botanicals into mainstream medicine. My message has always been about viewing each individual as unique and then considering a wide range of options available for treatment. This may involve herbs, but it may also include prescription medication, surgery, massage, acupuncture, or all of the above. I am not trying to force the integration of herbs into a health professional's practice; however, if there is evidence that an herb is effective for a particular condition, the herb should be considered alongside any other treatment option.

My strongest advocacy position right now is not that we teach alternative and complementary medicine in medical schools but that we bring humanities back into medical education. While stressing the importance of evidence-based, scientific medicine, it is imperative that we prepare health care providers for the real world of interacting with patients on a day-to-day basis.

By weaving the humanities throughout the educational process, the medical student, or practitioner, learns to integrate the emotional, spiritual, mental, and physical dimensions of the patient. While science can provide information about the best therapy for a given condition, it does little to help the practitioner answer questions such as, "how will I tell my wife I have cancer?" or "why is this happening to my child?"

Integrative medicine is, for me, a reemergence of viewing the patient as a whole person, someone with a rich history, culture, and set of beliefs. It is important to involve the patient in the decision-making process in both the treatment of a particular condition and also in living a life that focuses on prevention. When viewed in totality, we may explore botanicals, massage, diet, lifestyle, spirituality, and any other factors that may come into play based upon that individual's perception and beliefs.

Some of my patients are not interested in taking herbs at all. They prefer medications with solid evidence and prepared under rigorous control. Others prefer herbs as a friendlier alternative to pharmaceutical drugs. The practitioner must work with the patient and create a treatment protocol that is effective, while honoring the individual's personal preferences.

RM: Can you give me an example of how you treat a patient?

TLD: A classic example is the patient who comes into the office complaining of fatigue. She is not sleeping well, has had 2-3 colds over the past winter, and just doesn't feel like herself. After taking a history and performing a physical exam, appropriate studies are undertaken. Depending upon the individual presentation, we might check for anemia and hypothyroidism, and/or do a screen for a number of autoimmune or chronic illnesses. Everything comes back within the normal range. While this is 
reassuring, the last thing this patient wants to hear is: "Everything looks normal. See you in a year." That is often what happens as conventional physicians are trained to look to rule out physical disease. But, between the time the body is healthy and the time the body is sick, there is a period where people just don't feel good. It is after being told, "everything is fine" that a number of folks will go to the acupuncturist, the herbalist, or the naturopath in search of answers to the question: "Why don't I feel good?"

In my experience, sometimes the person's physical body has become the voice for problems that may be emotional, spiritual, or psychological in nature. There is an imbalance and the only way the imbalance can find expression is through the physical body.

My approach tends to be: Do the standard tests to make sure that there aren't any physical abnormalities that could be causing the symptoms. If the tests come back normal, I tell the patient that the good news is that, to the best of my medical knowledge; based upon the history, exam, and testing; there isn't anything seriously wrong with his or her body. Then I explain that the mind, body, and spirit are all connected and that, when there is an imbalance in one, the others are affected.

I ask the patient: "Tell me a little about your day. What is your workday like? Tell me why you aren't sleeping well? When is the last time you experienced absolute joy? Can you tell me about the last time you belly laughed like a 5-year-old?" Sometimes, these questions get the patient's tears going: "I can't remember the last time I belly laughed." "I don't think I have ever experienced real joy." Often, they are simply overwhelmed with life and all of its responsibilities.

This is why the therapeutic relationship is important. A relationship means we're going to continue to see each other; we're going to work together. "Let's figure out a place to start." This may start by getting the patient to sleep better again. If a person has a good night's sleep then the day is going to be a little bit better. There will be a little more energy to take a walk in the evening. . . a little less need to drink that extra cup of coffee in the afternoon. We might create a 3-week plan to help her sleep better and then have her return and go from there.

Many patients feel overwhelmed so I do not give them a "laundry list" of things to do. The last thing this individual needs, when he or she is already completely stressed out, is to have a long list of changes needed to make! For some patients, this simply contributes to a perceived sense of failure-one more thing one can't accomplish.

One recommendation I make to patients who are feeling overwhelmed is a 20-minute bath by candlelight, listening to soft music, and sipping a strong cup of chamomile tea (3-4 teabags to 1 cup of water). They should keep the lights and TV off, and then, after soaking in the bath, use the candles to light the way to bed. After 14 days of performing this evening ritual, many patients will report less muscle tension and better sleep.

Exercise is of great benefit for establishing well-being. It improves mood, enhances circulation, and increases endorphins. But it can be difficult to convince a sleep-deprived mother of three children, who is running nonstop all day long, that she

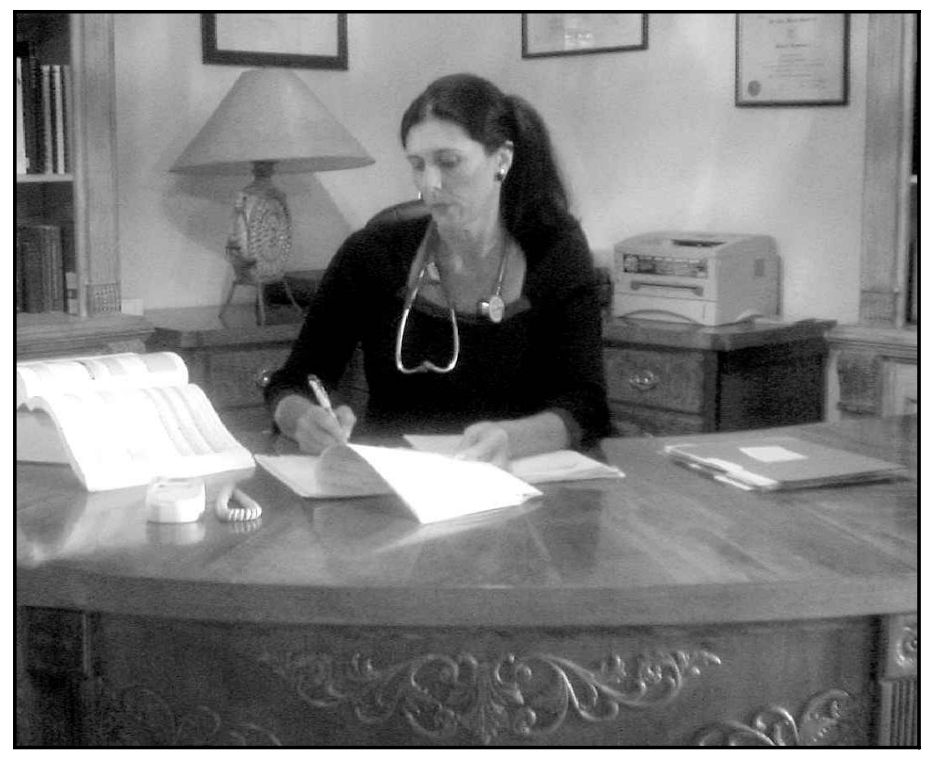

Tieraona Low Dog, M.D., director of the TreeHouse Center of Integrative Medicine.

needs to exercise! I generally start with relaxation and sleep and then add stretching, walking, etc.

My advice to practitioners is: Take it slow. Don't think you have to fix everything at once or even in a month or two. Start your patients slowly, and, this means for many patients, getting them to sleep better at night and helping them to see that they must be the \# 1 priority in their lives.

Other than chamomile, I didn't mention herbs did I?

RM: But, to return to herbs for a moment, the conventional model for use of herbs has been the German model, where one uses one specific, standardized herb, not in combination with other herbs. Please comment on this.

TLD: Yes, many practitioners have embraced the German monotherapy model and have said: "This is how herbalism is practiced," but, in truth, it only represents one model. It is a perfectly reasonable model and one that is well-suited to clinical testing. It has, in many ways, allowed more people to become more familiar with herbs and has led to increased research being conducted in the United States. What it doesn't do is accurately represent the actual practice of herbal medicine by a majority of practitioners-whether they are trained in Western, Chinese, or Ayurvedic herbal medicine.

\section{RM: What are some of your other concerns?}

TLD: I am concerned about the widespread, seemingly indiscriminate use of pharmaceuticals, particularly with children. It's staggering when you look at the number of children on Ritalin, the \$5-6 billion spent annually on antidepressants, and the millions of people who have had bypass surgery as they eat their hamburgers all the way to the surgeon's office.

It's staggering when one thinks that the tobacco companies are now advertising their good deeds as part of their settlement while, at the same time, targeting Hispanic teenagers. Now we 


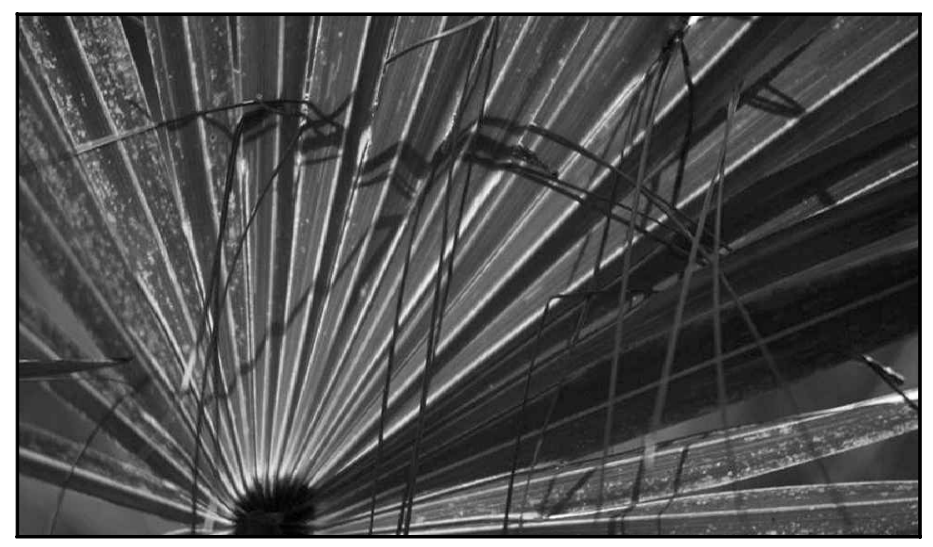

Saw palmetto (Serenoa repens).

have Jose and Josephina Camel in New Mexico selling cancer. Diabetes used to be a disease of old age. Old people got type 2 diabetes. Now we see it in children 12 or 13 years of age, especially amongst many Native American youth. Is this health care? Are we doing a good job?

At the present time, dietary supplements and botanicals constitute an industry worth more than $\$ 14$ billion; but, instead of getting to the root of our health problems and promoting wellness, many people are still looking for a "magic" pill. Consumers want to find a pill, preferably "natural" that will allow them to be healthy without changing anything in their lives. It won't work.

Pharmaceutical companies primarily drive medical research in this country. Herbs are natural substances and cannot be patented. This has led to a dearth of research in this country by herbal manufacturers and a misperception that herbs have no value because they have not been researched. The truth is, however, that a number of them have been researched in Europe and Asia. Some botanicals and dietary supplements have scientific evidence of benefit; others do not.

Saw palmetto [Serenoa serrulata and Serenoa repens] is a good example. A meta-analysis in the Journal of the American Medical Association* $^{*}$ and a review by the Cochrane Collaboration ${ }^{\dagger}$ have both found saw palmetto superior to placebo for the treatment of

*Wilt TJ, Ishani A, Stark G, MacDonald R. Lau J, Mulrow C. Saw palmetto extracts for treatment of benign prostatic hyperplasia: A systematic review. JAMA 1998;280:1604-1609; erratum in 1999;281:515.

$\dagger$ Wilt T, Ishani A, Mac Donald R. Serenoa repens for benign prostatic hyperplasia [Cochrane Review]. The Cochrane Library, Issue 3, 2003.

$\$$ McAlindon T. Glucosamine for osteoarthritis: Dawn of new era? Lancet 2001 27;357:247-248.

§Payne RB. Glucosamine sulphate and osteoarthritis. Lancet 2001; 357:1617; author reply by McAlindon T 2001;357:1618; erratum in 2001;358;1018. mild benign prostatic hyperplasia (enlarged prostate). The herb does not work as quickly as the prescription medication generally used for the condition but has the advantage of fewer sideeffects. However, if you have a limited income and it is a \$2 copay at the hospital formulary for the prescription and it is $\$ 30$ at the health food store for the herb, it isn't really a choice, is it? Saw palmetto is not covered because we view it as complementary and alternative medicine and there is no pharmaceutical company lobbying for it to be covered.

\section{RM: What about arthritis, which is fairly widespread?}

TLD: Glucosamine has been used for more than 30 years in Europe and has been clinically shown to be of benefit for some patients with osteoarthritis. It has two advantages over the medications commonly used for arthritis; it doesn't cause ulcers and it doesn't adversely affect the kidneys. A study published in The Lancet ${ }^{\ddagger}, \S$ showed that glucosamine may preserve the joint space, slowing the progression of the disease. If this is true, glucosamine will be the first disease modifying agent discovered so far for osteoarthritis. Given the evidence, safety profile and cost, it makes sense for a patient to try it for 2-3 months to see if it helps to relieve symptoms.

RM: Are there other herbs that practitioners might use for specific conditions?

TLD: I like garlic [Allium sativum]. It has been primarily studied for its benefits in lowering cholesterol but it was used for centuries as an antimicrobial. It used to be called "Russian penicillin" because it was so valued in poorer countries for its antiseptic properties.

Albert Schweitzer was said to have effectively treated many cases of dysentery with garlic. Louis Pasteur was the first to scientifically document its antiseptic properties. Japanese researchers have found that garlic, even when heated, is quite effective against the E. coli organism associated with food poisoning. Garlic has a mild cholesterol-lowering effect and may prevent the progression of atherosclerosis when consumed as part of a heart healthy diet and lifestyle. If you like garlic eat it. "Let thy food be thy medicine and thy medicine be thy food," as Hippocrates rightly said more than 2000 years ago.

\section{RM: What other herbs do you find to be beneficial?}

TLD: I find saw palmetto to be beneficial for enlarged prostate [as I noted before], especially in combination with pygeum [Prunus africanum]. Osha [Ligusticum porteri] is an herb that grows in the mountains of New Mexico that is very useful for colds, coughs, and minor congestion. Osha syrup is a favorite of mine. Echinacea [Echinacea spp.] tincture works great if you take enough of it, early enough, in the course of an upper respiratory infection. Chamomile is a great relaxant for the nerves and the gut especially in the young and the old. Lemonbalm [Melissa officinalis] is also lovely for lifting the mood and inducing a state of calm. Green or black tea [Camellia sinensis] works great for diarrhea. Topically 
applied, arnica [Arnica montana] has eased the pain of many a bruise. Ginger [Zingiber officinale] really does work for nausea.

On a deeper note, I believe that gardens themselves are very healing. To be surrounded by the exquisite beauty of nature is to experience a healing of the soul. Joseph Campbell said that it is in the garden that wonders are revealed. So true. The garden is one place where the sacred is made visible. We have the power within us, just like the seed, to grow.

I'm hoping for the greening of medicine, and I think plants are symbolic of that. They're one piece of it but if we just look to herbs as replacement drugs without doing anything else, we've missed this opportunity to really change our lives for the better. Paradise comes from the Persian word for garden. The plants themselves are transformative.

RM: Despite your love of natural healing remedies, I realize that you are an allopathic physician and have respect for standard practices. Please comment on this.

TLD: Of course. I am grateful for antibiotics, emergency medicine, skillful surgery, and all the other incredible gifts offered by conventional medicine. It would be very scary to wake up tomorrow and find that all prescription medications, hospitals, emergency care, nurses, doctors, lab technicians, and diagnostic equipment were gone. Imagine the consequences. When you find yourself at odds with the system, honestly imagine our world with all of it gone! Having said that, it is important to remember that there may be other approaches to some of the problems we are dealing with today. Many approaches are worthy of exploration and investigation.

RM: Being part Native American, do you use any Native American healing methods in your practice?

TLD: My approach is pretty eclectic. I have spent many years with people from different cultures: Lakota, Navajo, Zuni, Pueblo, Mexican-Americans in southern New Mexico, AfricanAmericans in the heart of the South, granny midwives of southern Appalachia, physicians, and nurses. My practice is really a compilation of the wisdom and magic of many healers. I have learned from Vietnamese and Korean healers through the study of Tae Kwon Do since 1977. Tae Kwon Do is a Korean Martial art, similar to Japanese Karate, with a greater emphasis placed on kicking and jumping kicks.

\section{RM: Has Tae Kwon Do been helpful to you?}

TLD: Tae Kwon Do is magical. There is no other word for it. More people should train in this art, especially children. Martial arts has been very powerful in my family. My son, a second degree black belt in Tae Kwon Do, found it very helpful for him in terms of channeling his energy and focus. It is powerful to watch your body transform itself right before your eyes. You take someone who can't even touch their toes and, over the many months of training, they metamorphosize until they are doing the splits, or kicking over their heads....

RM: I am impressed. Tell me a bit more.

TLD: Martial arts teach you that when you put your mind to

\section{How Safe is Black Cohosh?}

\section{Dr. Tieraona Low Dog's Integrative Approach to the Data}

Dr. Low Dog and two colleagues recently published a paper in Menopause regarding the safety of black cohosh (Cimicifuga racemosa), a remedy used by many women today to gain relief for women who are experiencing menopausal symptoms. This herb was traditionally used by Native Americans for assisting women in labor or for treating depression.

The paper, by Low Dog et al., was a safety review that examined black cohosh's use for patients who are unable to use conventional treatments for menopausal symptoms, such as estrogen replacement. The research team tapped an extensive database of literature on the herb, including all U.S. published literature about preclinical and clinical safety; foreign literature; Food and Drug Administration and World Health Organization adverse reports; various monographs and compendia; unpublished data from a major manufacturer; and historical anecdotal reports.

Collectively, analysis of the data revealed the following:

- Of 2800 patients using black cohosh, the incidence of adverse events was 5.4 percent.

- Of the reported adverse events, 97 percent were minor and did not cause patients to discontinue use of the herb.

- Any severe events that occurred were not the result of treatment with the herb.

Dr. Low Dog and her colleagues concluded that the effectiveness of black cohosh was dependent upon the preparation used and that the extracts that were included in the review were safe, especially isopropanolic preparations.

Source: Low Dog T, Powell KL, Weisman SM. Critical evaluation of the safety of Cimicifuga racemosa in menopause symptom relief. Menopause 2003;10:299-313.

something, when you are disciplined enough to keep going to class, even when you're tired and your body hurts, that you can do anything. You realize you have the ability to do anything. For myself, I went back to school and got my GED [General Education Diploma], which I don't think I would have done without Tae Kwon Do. This led me to college and then to medical school. All of my progress has been a direct result of my training in this ancient art form. It has given me immense confidence, direction and physical fitness. . . there is nothing more powerful.

\section{RM: How did you find your way to Tae Kwon Do?}

TLD: When my family moved to Virginia I was working as a leather craftsman. I enjoyed making things from leather and doing beadwork. One day, a Tae Kwon Do instructor asked me 


\section{To Contact Dr. Tieraona Low Dog}

Tieraona Low Dog, M.D.

Tree House Center of Integrative Medicine

Albuquerque, New Mexico

353 Loma Larga NW

Corrales, NM 87048

e-mail (preferred method of contact): lowdogmd@aol.com

to make some gymnastic mats for use in their classes. I handsewed these mats and took part of the payment in exchange for classes. I really didn't know what Tae Kwon Do was at the time.

\section{RM: What was it like?}

TLD: Tae Kwon Do classes were very intense and I was one of only a few women in the school. The classes were 90 minutes and exhausting. But within 2 months I was hooked. I lived and breathed Tae Kwon Do. I logged in roughly 600 classes per year, often taking classes 2-3 times per day. The training was of tremendous benefit to me and I became friends with the other students and their families. This was a pivotal experience for me and Tae Kwon Do remains an important part of my life today. I still love to jump, kick, stretch and punch; demanding of my body and spirit all that it has. And, like everyone else, I often feel tired or lazy, but the discipline of the training makes me dig in and keep going. My 9 year-old daughter takes Tae Kwon Do and she will say, "Mom, I am just too tired to go to class," and I will say, "I know, I know. Let's go."

RM: Apart from the good physical training, Tae Kwon Do is still a martial art, a means of defending oneself against attack. Have you ever had to defend yourself?

TLD: Just once in all these years. People with years of martial arts training are seldom bothered; most have this very gentle strength about them. Some "Americanized" martial arts schools are designed just for tournament fighting, tending to leave the "art" out of martial arts; sometimes these folks can be a little more arrogant and cocky. But with formal training, you learn to fight so you won't have to. It's mental, not just physical.

II Kitaro is a Japanese composer and performer of electronic instrumental music whose recordings are distributed in the United States by Domo Records, Los Angeles, California.
RM: How did this martial art get you into medicine?

TLD: I was teaching Tae Kwon Do at New Mexico State University, as part of the physical education department. As a result, I was part of the faculty and I didn't even have my high school diploma. I decided it was time to get that GED and from there it was just a natural progression to college and then medical school.

RM: Do you recommend Tae Kwon Do training to your friends or even to your patients?

TLD: Of course! [laughter] So many people need to exercise but they don't know what to do. I suggest that they try a good martial arts class. Martial arts training includes aerobic conditioning, as well as increasing your flexibility. One of the tenets of martial arts is "Inside like a lion, outside like a lamb." When you are strong and secure, you are not fearful. People who are fearful are often mean, because they are insecure. When you are secure in yourself and secure in your being, you can afford to be gentle and loving. You will never meet more gentle, loving people than these old martial arts masters.

RM: For some martial arts, meditation is a component. Is this true for you also?

TLD: To be honest with you, I am not a great meditater and never have been. I am a bit too "hyper" for that. However, I am very good at relaxing, and I enjoy stretching to soft music, such as Kitaro, II and doing deep breathing while I do my stretching. This is incredibly relaxing and I think my mind goes into a meditative state. But to sit for long periods in posture is not something I am especially good at. So it's good that there are lots and lots of ways to soothe your mind and senses. For some people, taking a bath by candlelight, with soft music in the background, can take them to very deep places of relaxation.

But, regardless of the method, one must take time to quiet one's mind. This is especially true for people who live in cities or who work in very crowded environments, since there is so much energy, people, noise, traffic, and other sensory bombardments.

RM: Dr. Low Dog, thank you so much for talking with us today.

TLD: I've had a delightful time talking with you. Thanks!

To order reprints of this article, write to or call: Karen Ballen, ALTERNATIVE \& COMPLEMENTARY THERAPIES, Mary Ann Liebert, Inc., 2 Madison Avenue, Larchmont, NY 10538-1961, (914) 834-3100. 